\title{
Optimal fourth order Weerakoon-Fernando type methods for multiple roots and their dynamics
}

\author{
Prem Bahadur Chand, Francisco I. Chicharro, Pankaj \\ Jain and Kriti Sethi
}

\begin{abstract}
In this paper, we present optimal fourth order methods for finding multiple roots of non-linear equations, where the multiplicity is known in advance. These methods are based on the third order method given by Weerakoon and Fernando for simple roots. The dynamical behavior of these methods around multiple roots is studied using basin of attraction in complex plane. We also present numerical examples to confirm our theoretical results.
\end{abstract}

Mathematics Subject Classification (2010). 65H05.

Keywords. Non-linear equations, Newton type method, multiple root, complex dynamics, fixed and critical points, basin of attraction.

\section{Introduction}

Solving non linear equations has always been an important task in all branches of science and engineering, in particular, in mathematical sciences. To find the exact roots of such equations is not always easy. In such situations, iterative methods are employed which give approximate roots. The task becomes more tedious if the desired root has multiplicity more than 1 . This paper is devoted to finding approximate roots of higher multiplicity.

The most widely used iterative method for finding a simple root of a non-linear equation $f(x)=0$ is the Newton method given by

$$
x_{n+1}=x_{n}-\frac{f\left(x_{n}\right)}{f^{\prime}\left(x_{n}\right)}
$$

This research was partially supported by both Ministerio de Ciencia, Innovación y Universidades and Generalitat Valenciana, Spain, under grants PGC-2018-095896-B-C22 and PROMETEO/2016/089, respectively. 
which is quadratically convergent. However, for multiple roots, this method is only linearly convergent. To retain the quadratic convergence of the method one of the ideas is to replace $f$ by $\frac{f}{f^{\prime}}$ so that (1.1) becomes

$$
x_{n+1}=x_{n}-\frac{f\left(x_{n}\right) f^{\prime}\left(x_{n}\right)}{f^{\prime}\left(x_{n}\right)^{2}-f\left(x_{n}\right) f^{\prime \prime}\left(x_{n}\right)} .
$$

This method is known to be of order 2 , but at the expense of involving second derivative. Alternatively, if the multiplicity of the root is known, say $m$, then (1.1) is modified as

$$
x_{n+1}=x_{n}-m \frac{f\left(x_{n}\right)}{f^{\prime}\left(x_{n}\right)}
$$

which is again known to be of order 2 and yet does not involve second derivative.

With the aim of increasing the order of Newton method, Weerakoon and Fernando [13] gave the following method

$$
\begin{aligned}
y_{n} & =x_{n}-\frac{f\left(x_{n}\right)}{f^{\prime}\left(x_{n}\right)}, \\
x_{n+1} & =x_{n}-\frac{2 f\left(x_{n}\right)}{f^{\prime}\left(x_{n}\right)+f^{\prime}\left(y_{n}\right)},
\end{aligned}
$$

for finding simple roots. This is a cubically convergent method. It can be checked that for multiple roots, the method (1.3) is only linearly convergent. The first aim of this paper is to derive a method of the type (1.3) for multiple roots which is of order 3 .

In [7], Kung and Traub conjectured that the method is optimal if its order is $p=2^{n-1}$, where $n$ is the number of function evaluations per iteration. In that sense, neither the method (1.3) nor the one we propose is optimal . In this paper, we improve our own method having order 4 and requires 3 functions evaluations per iteration which makes the method optimal. We also present another optimal fourth order method involving weight functions.

Let us mention that recently, some different fourth order methods have been obtained for finding multiple roots of non-linear equations. In this direction, Chun and Neta obtained the following method:

$$
x_{n+1}=x_{n}-\frac{2 m^{2} f\left(x_{n}\right) f^{\prime \prime}\left(x_{n}\right)}{m(m-3) f\left(x_{n}\right) f^{\prime \prime}\left(x_{n}\right)+(m-1)^{2} f^{\prime}\left(x_{n}\right)^{2}} \frac{f\left(x_{n}\right)}{f^{\prime}\left(x_{n}\right)} .
$$

Also, Zhou and Liu in [8], proposed a fourth order method for obtaining multiple roots

$$
\begin{aligned}
y_{n} & =x_{n}-m \frac{f\left(x_{n}\right)}{f^{\prime}\left(x_{n}\right)}, \\
w_{n} & =\left(\frac{f^{\prime \prime}\left(y_{n}\right)}{f^{\prime}\left(x_{n}\right)}\right)^{1 /(m-1)}, \\
G\left(w_{n}\right) & =w_{n}+\frac{2 m}{m-1} w_{n}^{2}, \\
x_{n+1} & =y_{n}-m G\left(w_{n}\right) \frac{f\left(x_{n}\right)}{f^{\prime}\left(x_{n}\right)} .
\end{aligned}
$$

In 2010, Sharma and Sharma [10], presented a fourth order method for computing multiple roots which is based on Jaratt's method given by:

$$
x_{n+1}=x_{n}-a_{1} w_{1}\left(x_{n}\right)-a_{2} w_{2}\left(x_{n}\right)-a_{3} \frac{w_{2}^{2}\left(x_{n}\right)}{w_{1}\left(x_{n}\right)},
$$


where

$$
\begin{gathered}
w_{1}\left(x_{n}\right)=\frac{f\left(x_{n}\right)}{f^{\prime}\left(x_{n}\right)}, \quad w_{2}\left(x_{n}\right)=\frac{f\left(x_{n}\right)}{f^{\prime}\left(y_{n}\right)}, \quad y_{n}=x_{n}+\beta w_{1}\left(x_{n}\right), \\
a_{1}=\frac{1}{8} m\left(m^{3}-4 m+8\right), \quad a_{2}=\frac{1}{4}(-m)(m-1)(m+2)^{2}\left(\frac{m}{m+2}\right)^{m}, \\
a_{3}=\frac{1}{8} m(m+2)^{3}\left(\frac{m}{m+2}\right)^{2 m}, \quad \beta=-\frac{2 m}{m+2} .
\end{gathered}
$$

We shall compare the methods (1.4), (1.5), and (1.6) with the new methods obtained in this paper.

The novelty of this paper is that we shall study the dynamical behaviour of our methods with the help of basin of attraction. A lot of literature is available concerning the study of dynamics of Newton type method for simple roots. However, for multiple roots such study is very rarely seen.

\section{Development of Methods and Convergence Analysis}

Recall that Weerakoon and Fernando's method (1.3) has cubic convergence for simple roots. In case of multiple roots, this method looses its order of convergence and its error equation, in this case, can be obtained as

$$
e_{n+1}=\left(1-\frac{2(m-1)}{m\left(m\left(\frac{m-1}{m}\right)^{m}+m-1\right)}\right) e_{n}+O\left(e_{n}^{2}\right)
$$

which indicates that this method is linearly convergent. To this end, we propose the following method:

$$
\begin{aligned}
y_{n} & =x_{n}-\frac{2 m}{m+2} \frac{f\left(x_{n}\right)}{f^{\prime}\left(x_{n}\right)}, \\
x_{n+1} & =x_{n}-\frac{2 f\left(x_{n}\right)}{a_{1} f^{\prime}\left(x_{n}\right)+a_{2} f^{\prime}\left(y_{n}\right)},
\end{aligned}
$$

where $a_{1}$ and $a_{2}$ are the parameters to be chosen suitably.

Theorem 2.1. Let $f$ be a sufficiently differentiable function and $\alpha$ be a root of $f(x)=0$ of multiplicity $m$. If $x_{0}$ is sufficiently close to $\alpha$, then the method (2.1) has order of convergence three provided

$$
a_{1}=-1+\frac{2}{m}-\frac{m}{2}, \quad a_{2}=\frac{1}{2} m^{1-m}(2+m)^{m} .
$$

Proof. Let $e_{n}$ and $d_{n}$ be the errors, respectively, in $x_{n}$ and $y_{n}$. Using Taylor series, we have

$$
f\left(x_{n}\right)=\frac{f^{(m)}(\alpha)}{m !} e_{n}^{m}\left[1+C_{1} e_{n}+C_{2} e_{n}^{2}+C_{3} e_{n}^{3}+C_{4} e_{n}^{4}+O\left(e_{n}^{5}\right)\right],
$$

and

$$
\begin{aligned}
f^{\prime}\left(x_{n}\right) & =\frac{f^{(m)}(\alpha)}{(m) !} e_{n}^{m-1}\left[m+(1+m) C_{1} e_{n}+(2+m) C_{2} e_{n}^{2}\right. \\
& \left.+(3+m) C_{3} e_{n}^{3}+(4+m) C_{4} e_{n}^{4}+O\left(e_{n}^{5}\right)\right]
\end{aligned}
$$


where $C_{j}=\frac{m !}{(m+j) !} \frac{f^{(m+j)}(\alpha)}{f^{(m)}(\alpha)}, j=1,2, \ldots$ Therefore, the first step of $(2.1)$ gives

$$
\begin{aligned}
d_{n} & =m \frac{e_{n}}{2+m}+\frac{2 C_{1}}{m(m+2)} e_{n}^{2}+\frac{\left(-2(1+m) C_{1}^{2}+4 m C_{2}\right)}{m^{2}(2+m)} e_{n}^{3} \\
& +\frac{2\left((1+m)^{2} C_{1}^{3}-m(4+3 m) C_{1} C_{2}+3 m^{2} C_{3}\right)}{m^{3}(2+m)}+O\left(e_{n}^{5}\right)
\end{aligned}
$$

Using (2.4) and Taylor series, we get

$$
\begin{aligned}
f^{\prime}\left(y_{n}\right) & =\frac{f^{(m)}(\alpha)}{(m) !} d_{n}^{m-1}\left[m+(1+m) C_{1} d_{n}+(2+m) C_{2} d_{n}^{2}\right. \\
& \left.+(3+m) C_{3} d_{n}^{3}+(4+m) C_{4} d_{n}^{4}+O\left(d_{n}^{5}\right)\right],
\end{aligned}
$$

so that by using (2.2), (2.3) and (2.5) in (2.1), we obtain the error equation of (2.1) as

$$
\begin{aligned}
e_{n+1} & =e_{n}\left(1-\frac{2}{a_{1} m+a_{2} m^{m}(m+2)^{1-m}}\right) \\
& +\frac{2 c_{1} e_{n}^{2}\left(a_{1} m^{2}+a_{2}(m(m+2)-4)\left(\frac{m}{m+2}\right)^{m}\right)}{m^{2}\left(a_{1} m+a_{2} m^{m}(m+2)^{1-m}\right)^{2}}-A e_{n}^{3}+O\left(e_{n}^{4}\right),
\end{aligned}
$$

where

$$
\begin{aligned}
A & =\frac{1}{m^{3}\left(a_{1} m+a_{2} m^{m}(m+2)^{1-m}\right)^{3}} 2\left(a_{1}^{2} m^{3}\left(C_{1}^{2}(m+1)-2 C_{2} m\right)\right. \\
& +2 a_{1} a_{2}\left(\frac{m}{m+2}\right)^{m}\left(C_{1}^{2}\left(m^{4}+3 m^{3}-2 m-4\right)-2 C_{2} m^{2}(m(m+2)-2)\right) \\
& +a_{2}^{2}\left(\frac{m}{m+2}\right)^{2 m}\left(C_{1}^{2}(m(m(m+1)(m+4)-8)-16)\right. \\
& \left.\left.-2 C_{2} m\left(m^{2}(m+4)-8\right)\right)\right) .
\end{aligned}
$$

Thus, for

$$
a_{1}=-1+\frac{2}{m}-\frac{m}{2}, \quad a_{2}=\frac{1}{2} m^{1-m}(2+m)^{m},
$$

then (2.6) reduces to

$$
e_{n+1}=\frac{C_{1}^{2}(m-2)}{m^{3}} e_{n}^{3}+O\left(e_{n}^{4}\right)
$$

and the assertion follows.

Remark 2.2. Observe that, if we put $m=2$ in (2.8), the coefficient of $e_{n}^{3}$ becomes zero and therefore, the method (2.1) becomes of order four. In that case $a_{1}=-1$ and $a_{2}=4$. The corresponding fourth order method reads as

$$
\begin{aligned}
y_{n} & =x_{n}-\frac{f\left(x_{n}\right)}{f^{\prime}\left(x_{n}\right)}, \\
x_{n+1} & =x_{n}-\frac{f\left(x_{n}\right)}{4 f^{\prime}\left(y_{n}\right)-f\left(x_{n}\right)} .
\end{aligned}
$$

In the sense of Kung Traub, the method (2.9) is optimal as it requires 3 functions evaluation per iterations.

In the view of Remark 2.2, the method (2.9) is fourth order and optimal. However, it has a limitation that the multiplicity of the targeted root should be 2. By using weight functions, we modify (2.1) (and consequently (2.9)) 
and propose the following method which is fourth order optimal method for any multiplicity $m$ :

$$
\begin{aligned}
y_{n} & =x_{n}-\frac{2 m}{m+2} \frac{f\left(x_{n}\right)}{f^{\prime}\left(x_{n}\right)}, \\
x_{n+1} & =x_{n}-\left(\frac{2 b_{2} f\left(x_{n}\right)}{b_{1} f^{\prime}\left(x_{n}\right)+f^{\prime}\left(y_{n}\right)}\right)\left(1+b_{3} \frac{f^{\prime}\left(y_{n}\right)}{f^{\prime}\left(x_{n}\right)}\right),
\end{aligned}
$$

where $b_{1}, b_{2}$ and $b_{3}$ are the parameters to be chosen suitably. The following theorem proves the convergence of the method (2.10).

Theorem 2.3. Let $f$ be a sufficiently differentiable function in a neighbourhood of $\alpha$ which is a multiple root of $f(x)=0$ of multiplicity $m$. If $x_{0}$ is sufficiently close to $\alpha$ then the method (2.10) is of order four if

$$
b_{1}=-\mu^{m}, \quad b_{2}=\frac{1}{4} m^{2} \mu^{m}, \quad b_{3}=-\frac{(m-2) \mu^{-m}}{m},
$$

where $\mu=\frac{m}{m+2}$.

Proof. Using Taylor's expansion around $\alpha,(2.2),(2.3)$ and (2.5), we obtain the error equation of the method (2.10). The error equation is given by

$$
e_{n+1}=A_{1} e_{n}+A_{2} e_{n}^{2}+A_{3} e_{n}^{3}+A_{4} e_{n}^{4}+O\left(e_{n}^{5}\right),
$$

where

$$
\begin{aligned}
A_{1} & =1-\frac{2 b_{2}\left(2 b_{3} \mu^{m}+b_{3} m \mu^{m}+m\right)}{m_{M} M}, \\
A_{2} & =\frac{2 b_{2} C_{1}\left[4 b_{3} M \mu^{m} M+m\left(b_{1} m^{2}+\left(m^{2}+2 m-4\right) \mu^{m}\right)\left(b_{3} \mu^{m-1}+1\right)\right]}{m^{3} M^{2}} \\
A_{3} & =\frac{2 b_{2}}{m^{5} M^{3}}\left[-4 b_{3} \mu^{m} M C_{1}^{2}\left(b_{1} m^{2}+\left(m^{2}+2 m-4\right) \mu^{m}\right)-4 b_{3} \mu^{m} M^{2}\right. \\
& \times\left(C_{1}^{2}\left(m^{2}+2\right)-2 C_{2} m^{2}\right)-m\left(2 b_{3} \mu^{m}+b_{3} m \mu^{m}+m\right) \\
& \times\left(\left[b_{1}^{2} m^{3}(m+1)+2 b_{1}\left(m^{4}+3 m^{3}-2 m-4\right) \mu^{m}\right.\right. \\
& \left.+\mu^{2 m}\left(m^{4}+5 m^{3}+4 m^{2}-8 m-16\right)\right] C_{1}^{2} \\
& \left.\left.-2 m\left(b_{1}^{2} m^{3}+2 b_{1} m\left(m^{2}+2 m-2\right) \mu^{m}+\mu^{2 m}\left(m^{3}+4 m^{2}-8\right)\right) C_{2}\right)\right] \\
A_{4} & =\frac{2 b_{2}}{3 m^{7} M^{4}}\left[1 2 b _ { 3 } \mu ^ { m } M ^ { 2 } ( b _ { 1 } m ^ { 2 } + \mu ^ { m } ( - 4 + 2 m + m ^ { 2 } ) ) C _ { 1 } \left(C_{1}^{2}\left(m^{2}+2\right)\right.\right. \\
& \left.-2 C_{2} m^{2}\right)+12 C_{1} b_{3} m^{m+1}(m+2)^{-m} M\left(\left(b_{1}^{2} m^{3}(m+1)+2 b_{1}\right.\right. \\
& \left.\times\left(m^{4}+3 m^{3}-2 m-4\right) \mu^{m}+\mu^{2 m}\left(m^{4}+5 m^{3}+4 m^{2}-8 m-16\right)\right) C_{1}^{2} \\
& \left.-2 m\left(b_{1}^{2} m^{3}+2 b_{1} m\left(m^{2}+2 m-2\right) \mu^{m}+\mu^{2 m}\left(m^{3}+4 m^{2}-8\right)\right) C_{2}\right) \\
& +8 b_{3} m^{m}(m+2)^{-m-2} M^{3}\left(C_{1}^{3}(m+2)^{2}\left(m^{4}-m^{3}+5 m^{2}+m+6\right)\right. \\
& \left.-3 C_{1} C_{2} m^{2}(m+2)^{2}\left(m^{2}+4\right)+3 C_{3} m^{4}\left(m^{2}+6 m+6\right)\right)+\frac{1}{(m+2)^{2}} \\
& \times m\left(2 b_{3} \mu^{m}+b_{3} m \mu^{m}+m\right)(m+2)^{2}\left(3 b_{1}^{3} m^{5}(m+1)^{2}+b_{1}^{2} m \mu^{m}\right. \\
& \times\left(9 m^{6}+36 m^{5}+37 m^{4}+2 m^{3}-52 m^{2}-32 m-48\right)+b_{1} m \mu^{2 m} \\
& \times\left(9 m^{6}+54 m^{5}+101 m^{4}+44 m^{3}-132 m^{2}-176 m-176\right)+\mu^{3 m} \\
& \left.\times\left(3 m^{7}+24 m^{6}+67 m^{5}+66 m^{4}-64 m^{3}-184 m^{2}-144 m-32\right)\right) C_{1}^{3} \\
& -3 m^{2}(m+2)^{2}\left(b_{1}^{3} m^{4}(3 m+4)+b_{1}^{2} m\left(9 m^{4}+30 m^{3}+16 m^{2}\right.\right. \\
& \left.-16 m^{2}-32\right) \mu^{m}+\mu^{3 m}\left(3 m^{5}+22 m^{4}+52 m^{3}+24 m^{2}-96 m-128\right) \\
& \left.+b_{1} \mu^{2 m}\left(9 m^{5}+48 m^{4}+68 m^{3}-16 m^{2}-128 m-64\right)\right) C_{1} C_{2} \\
& +3 C_{3} m^{3} M^{2}\left(3 b_{1} m^{2}(m+2)^{2}\right. \\
& \left.\left.+\left(3 m^{4}+18 m^{3}+28 m^{2}-24 m-48\right) \mu^{m}\right)\right] \\
& =b_{1} m
\end{aligned}
$$

and $M=b_{1} m+\mu^{m}(2+m)$. Now, in order to get the order of convergence four, we must have $A_{1}=A_{2}=A_{3}=0$, which is true when $b_{1}, b_{2}, b_{3}$ are 
given by (2.11). Consequently (2.12) reduces to

$$
\begin{aligned}
e_{n+1} & =\frac{\left((2+m) C_{1}\left((-2+m(2+m(2+m))) C_{1}^{2}-3 m^{3} C_{2}\right)+3 m^{5} C_{3}\right)}{3 m^{4}(2+m)^{2}} e_{n}^{4} \\
& +O\left(e_{n}^{5}\right),
\end{aligned}
$$

and the assertion follows.

\section{Numerical Results}

In this section, we compare our methods (2.1) and (2.10) with Newton's method (MN), Chun and Neta's method (MCN), Liu et. al method (ML) and Sharma and Sharma method (MSS) referenced in Section 1. All computations have been done using MATHEMATICA. The functions used to test the methods and compare the results are given in Table 1, where the multiplicity $m$ and the root $\alpha$ are pointed.

TABLE 1. Test functions for analyzing the iterative methods.

\begin{tabular}{|l|c|c|}
\hline$f(x)$ & $m$ & $\alpha$ \\
\hline$f_{1}=\left(x^{2}-e^{x}-3 x+2\right)^{6}$ & 6 & 0.2575302854398607 \\
\hline$f_{2}=\left(x^{3}+4 x^{2}-10\right)^{3}$ & 3 & 1.3652300134140968 \\
\hline$f_{3}=(1+\cos x)\left(e^{x}-2\right)^{2}$ & 2 & 0.6931471805599453 \\
\hline$f_{4}=\left(e^{x^{2}+7 x-30}-1\right)^{6}$ & 6 & 3.0000000000000000 \\
\hline$f_{5}=(\log x+\sqrt{x}-5)^{5}$ & 5 & 8.3094326942315717 \\
\hline
\end{tabular}

Tables 2-3 show the performance of the aforementioned methods. It includes the number of iterations $(n)$ required such that $|f(x)|<10^{-30}$. In Tables 2-3, $a(b)$ denotes $a \times 10^{-b}$.

TABLE 2. Numerical results of the methods MN, MCN and (2.1) applied on the test functions of Table 1.

\begin{tabular}{|c|c|c|c|c|c|c|c|}
\hline & & \multicolumn{2}{|c|}{ MN } & \multicolumn{2}{|c|}{ MCN } & \multicolumn{2}{|c|}{$(2.1)$} \\
\hline$f(x)$ & $x_{0}$ & $n$ & $f\left(x_{n}\right)$ & $n$ & $f\left(x_{n}\right)$ & $n$ & $f\left(x_{n}\right)$ \\
\hline$f_{1}$ & -7 & 5 & $1.50(37)$ & 4 & $2.12(88)$ & 3 & $3.19(51)$ \\
& 1.4 & 3 & $1.13(37)$ & 3 & $7.58(76)$ & 2 & $1.88(31)$ \\
\hline$f_{2}$ & 3.5 & 6 & $7.00(43)$ & 4 & $7.00(43)$ & 3 & $2.84(34)$ \\
& 0.4 & 7 & 0 & 16 & $4.48(44)$ & 12 & $5.6(42)$ \\
\hline$f_{3}$ & 0.2 & 5 & 0 & 4 & 0 & 3 & 0 \\
& 1.5 & 5 & 0 & 4 & 0 & 3 & 0 \\
\hline$f_{4}$ & 3.2 & 6 & $8.08(39)$ & 4 & $1.95(61)$ & 3 & $4.41(75)$ \\
& 4 & 18 & $5.92(57)$ & 11 & $6.00(81)$ & 6 & $2.83(52)$ \\
\hline$f_{5}$ & 1.5 & 5 & $3.39(56)$ & 3 & $1.77(74)$ & 4 & $8.13(68)$ \\
& 10 & 3 & $4.39(43)$ & 2 & $9.51(64)$ & 2 & $2.29(54)$ \\
\hline
\end{tabular}


TABLE 3. Numerical results of the methods ML, MSS and (2.10) applied on the test functions of Table 1.

\begin{tabular}{|c|c|c|c|c|c|c|c|}
\hline & & \multicolumn{2}{|c|}{ ML } & \multicolumn{2}{|c|}{ MSS } & \multicolumn{2}{c|}{$(2.10)$} \\
\hline$f(x)$ & $x_{0}$ & $n$ & $f\left(x_{n}\right)$ & $n$ & $f\left(x_{n}\right)$ & $n$ & $f\left(x_{n}\right)$ \\
\hline$f_{1}$ & -7 & 3 & $1.49(48)$ & 3 & $1.52(76)$ & 3 & $1.14(84)$ \\
& 1.4 & 2 & $5.9(65)$ & 2 & $9.67(41)$ & 2 & $1.17(40)$ \\
\hline$f_{2}$ & 3.5 & 4 & 0 & 3 & $4.34(35)$ & 3 & $1.25(36)$ \\
& 0.4 & 16 & 0 & 12 & $1.23(31)$ & 5 & $3.59(43)$ \\
\hline$f_{3}$ & 0.2 & 4 & 0 & 3 & $1.39(30)$ & 3 & 0 \\
& 1.5 & 3 & 0 & 3 & $1.39(30)$ & 3 & 0 \\
\hline$f_{4}$ & 3.2 & 4 & $4.29(64)$ & 3 & $2.16(33)$ & 3 & $8.72(35)$ \\
& 4 & 11 & $2.42(30)$ & 9 & $2.12(33)$ & 9 & $1.74(40)$ \\
\hline$f_{5}$ & 1.5 & 3 & $9.86(64)$ & 2 & $1.51(48)$ & 2 & $1.44(50)$ \\
& 10 & 3 & 0 & 2 & $2.90(68)$ & 2 & $1.77(74)$ \\
\hline
\end{tabular}

From the tables 2-3, we can conclude that our methods (2.1) and (2.10) are competitive with the other methods in terms of number of iterations required.

\section{Dynamics of the Methods}

In this section, we study the dynamical behavior of the methods. In particular, we analyze the fixed points, critical points, basins of attraction and stability of the methods presented in this paper. For this, we apply the methods on complex polynomials $p(z)$ with degrees two and three having different multiplicities. It is well known that the fixed points and the critical points of any method play important role in the understanding of the dynamics of the corresponding method. We study the affect of order, degree of the polynomial and multiplicity on the number of extraneous points. Further, stability of the method is shown visually with the help of basins of attraction of the attracting fixed points. For more details of the complex dynamics of rational functions (or operators), one may refer to [1], [2], [3], [6] and references therein.

\subsection{Some basics}

Let $\hat{\mathbb{C}}=\mathbb{C} \cup(\infty)$ denote the extended complex plane. Let $p: \hat{\mathbb{C}} \rightarrow \hat{\mathbb{C}}$ be a function. A point $z_{0} \in \hat{\mathbb{C}}$ is called a fixed point of $p$ if $p\left(z_{0}\right)=z_{0}$. A fixed point $z_{0}$ of $p(z)$ is called attracting, super-attracting or repelling if, respectively, $0<\left|p^{\prime}\left(z_{0}\right)\right|<1, p^{\prime}\left(z_{0}\right)=0$ or $\left|p^{\prime}\left(z_{0}\right)\right|>1$. It is noted that $z=\infty$ is a super-attracting fixed point for any polynomial with degree $n \geq 2$.

Orbit of a point $z_{0} \in \hat{\mathbb{C}}$ of the mapping $p(z)$ is given by

$$
\operatorname{Orbit}\left(z_{0}\right)=\left\{z_{o}, p\left(z_{0}\right), p^{2}\left(z_{0}\right), \ldots \ldots\right\}
$$


The basin of attraction of an attracting (or super-attracting) fixed point $z_{0}$ of $p(z)$ is the set of all points whose orbits converge to $z_{0}$.

Consider for example the mapping $p(z)=z^{2}$. It can be checked that 0 and $\infty$ are super-attracting fixed points while 1 is a repelling fixed point for $p(z)$. Also, the basins of attraction for 0 and $\infty$ are, respectively $\{z:|z|<$ $1\}$ and $\{z:|z|>1\}$.

Corresponding to the function $p(z)$, define a transform

$$
M_{p}(z)=z-\phi_{p}(z),
$$

where $\phi_{p}(z)$ is such that $p(z)=0 \Rightarrow \phi_{p}(z)=0$. When $\phi_{p}(z)=\frac{p(z)}{p^{\prime}(z)}$, then the corresponding transform $M_{p}(z)$ is the well known Newton's transform. Clearly, the roots of $p(z)=0$ are the fixed points of $M_{p}(z)$. However, there may be fixed points of $M_{p}(z)$ which need not be the roots of $p(z)=0$. Such points are called extraneous fixed points. Consider, for example, $p(z)=$ $z^{2}-3 z+2$ and

$$
M_{p}(z)=z-\frac{z^{2}-3 z+2}{2 z-3}\left(z^{2}-3 z+3\right) .
$$

Here $z=1,2$ are the roots of $p(z)=0$ and therefore fixed points of $M_{p}(z)$. The points $z=\frac{3 \pm i \sqrt{3}}{2}$ are not the roots of $p(z)=0$ but are fixed points of $M_{p}(z)$ and therefore are the extraneous fixed points of $M_{p}(z)$.

\subsection{Fixed and critical points of the methods}

Let $p(z)$ be a polynomial having multiple roots defined on $\hat{\mathbb{C}}$. Corresponding to the methods $(2.2)$ and $(2.10)$, we define the operators $M_{3}(z), M_{4}(z) \in \hat{\mathbb{C}}$ as follows:

$$
\begin{aligned}
y(z) & =z-\frac{2 m}{m+2} \frac{p(z)}{p^{\prime}(z)}, \\
M_{3}(z) & =z-\frac{2 p(z)}{a_{1} p^{\prime}(z)+a_{2} p^{\prime}(y(z))}, \\
M_{4}(z) & =z-\frac{2 b_{2} p(z)}{b_{1} p^{\prime}(z)+p^{\prime}(y(z))}\left(1+b_{3} \frac{p^{\prime}(y(z))}{p^{\prime}(z)}\right) .
\end{aligned}
$$

From now on, we are analyzing the dynamical behavior of these methods when they are applied on two polynomials of second degree and two polynomials of third degree, both of them with multiplicities 3 and 4 .

4.2.1. Dynamical analysis of the method $M_{3}(z)$. When the polynomial $p_{1}(z)=$ $\left(z^{2}+1\right)^{3}$ is applied on $M_{3}(z)$, the resulting fixed point operator is

$$
M_{31}(z)=\left.M_{3}(z)\right|_{p_{1}(z)}=\frac{z+28 z^{3}+278 z^{5}-49 z^{7}}{1+28 z^{2}+128 z^{4}-199 z^{6}} .
$$

Solving $M_{31}(z)=z$ results in three different fixed points: $z_{1}^{*}=-i$ and $z_{2}^{*}=i$ are the roots of the polynomial $p_{1}(z)$, but $z_{3}^{F}=0$ is a strange fixed point. Both roots of the polynomial are superattracting fixed points, and $z_{3}^{F}$ is an indifferent fixed point, since $\left|M_{31}^{\prime}\left(z_{3}^{F}\right)\right|=1$. Moreover, $z_{\infty}^{*}=\infty$ is an attracting fixed point. 
The critical points are obtained from solving $M_{31}^{\prime}(z)=0$. There are 10 different critical points: $z_{1,2}^{*}, z_{3-6}^{C}= \pm 0.0793 \pm i 0.1556, z_{7,8}^{C}= \pm 1.2809$, and $z_{9,10}^{C}= \pm 0.2593$. Let us remark that only $z_{1,2}^{*}$ match with the roots of $p_{1}(z)$, so the number of free critical points is 8 .

Applying $p_{2}(z)=\left(z^{2}+1\right)^{4}$ on $M_{3}(z)$ results in the fixed point operator

$$
M_{32}(z)=\left.M_{3}(z)\right|_{p_{2}(z)}=\frac{z\left(1+70 z^{2}+1500 z^{4}+13162 z^{6}-2093 z^{8}\right)}{1+70 z^{2}+1500 z^{4}+6250 z^{6}-9005 z^{8}} .
$$

The fixed points match with those of $M_{32}(z)$. Regarding to the critical points, the number of points has increased to $14: z_{1,2}^{*}, z_{3,4}^{C}= \pm i 1.4423, z_{5,6}^{C}=$ $\pm i 0.2331, z_{7,8}^{C}= \pm i 0.1239, z_{9,10}^{C}= \pm i 0.1854$ and $z_{11-14}= \pm 0.0968 \pm i 0.1430$. Note that there are 12 free critical points.

When $p_{3}(z)$ is applied over $M_{3}(z)$, the fixed point operator gets into

$$
M_{33}(z)=\left.M_{3}(z)\right|_{p_{3}(z)}=\frac{N_{19}(z)}{D_{18}(z)},
$$

where $N_{19}(z)$ and $D_{18}(z)$ stand for a numerator and denominator of degrees 19 and 18, respectively. The fixed points of $M_{33}(z)$ are $4: z_{1,2}^{*}=-\frac{1}{2} \pm i \frac{\sqrt{3}}{2}$ and $z_{3}^{*}=1$, that agree with the roots of $p_{3}(z)$, and $z_{4}^{F}=0$. As usual, the three roots are superattracting fixed points. The evaluation of $\left|M_{33}^{\prime}\left(z_{4}^{F}\right)\right|$ points out that $z_{4}^{F}$ is an indifferent fixed point. In addition, $z_{\infty}^{*}=\infty$ is an attracting fixed point. In this case, the number of critical points is 30 . Every one of them, but the roots of $p_{3}(z)$, are critical points.

Finally, we evaluate the application of $p_{4}(z)$ on $M_{3}(z)$, whose fixed point operator is

$$
M_{34}(z)=\left.M_{3}(z)\right|_{p_{4}(z)}=\frac{N_{25}(z)}{D_{24}(z)},
$$

where, as mentioned in the $p_{3}(z)$ case, $N_{25}$ and $D_{24}$ are polynomials of degrees 25 and 24, respectively. The behavior of the four fixed points match with those of $M_{33}(z)$. However, there are 42 critical points, 39 of them satisfying the definition of free critical point.

Table 4 gathers the information related to the fixed and critical points of the methods $M_{31}(z), M_{32}(z), M_{33}(z)$ and $M_{34}(z)$.

TABLE 4. Fixed and critical points of $M_{3}(z)$ on different polynomials

\begin{tabular}{|c|c|c|c|c|}
\hline$p(z)$ & $M(z)$ & $\begin{array}{c}\text { Number of } \\
\text { roots }\end{array}$ & $\begin{array}{c}\text { Number of strange } \\
\text { fixed points }\end{array}$ & $\begin{array}{c}\text { Number of free } \\
\text { critical points }\end{array}$ \\
\hline$p_{1}(z)=\left(z^{2}+1\right)^{3}$ & $M_{31}(z)$ & 2 & 1 & 8 \\
$p_{2}(z)=\left(z^{2}+1\right)^{4}$ & $M_{32}(z)$ & 2 & 1 & 12 \\
$p_{3}(z)=\left(z^{3}-1\right)^{3}$ & $M_{33}(z)$ & 3 & 1 & 30 \\
$p_{4}(z)=\left(z^{3}-1\right)^{4}$ & $M_{34}(z)$ & 3 & 1 & 42 \\
\hline
\end{tabular}

Figure 1 represents the dynamical planes of the methods. These planes have been generated with MatlabR2017b ${ }^{\complement}$, following the guidelines of [4]. 
The initial guess are a set of $500 \times 500$ points in the region $-3<\Re\{z\}<3$, $-3<\Im\{z\}<3$. Each initial guess is iterated over the fixed point operator until the distance between the value and an attracting fixed point is closer than $10^{-3}$, or the number of iterations has reached the value 80 . Each attracting fixed point is mapped with a different color. Once the successive iterations of an initial guess has reached an attracting fixed point, the initial guess is represented with the color of the corresponding root. The map of colors for $p_{1,2}(z)$ is orange for $z_{1}^{*}=-i$ and blue for $z_{2}^{*}=i$, while the map for $p_{3,4}(z)$ is orange, blue and green for the roots $z_{1}^{*}=-\frac{1}{2}+i \frac{\sqrt{3}}{2}, z_{2}^{*}=-\frac{1}{2}-i \frac{\sqrt{3}}{2}$ and $z_{3}^{*}=1$, respectively. We have also included a color mapping for the fixed points 0 and $\infty$, whose corresponding colors are grey and purple, respectively. Every fixed point is plotted with a white circle in the complex plane. Moreover, the amount of iterations needed to reach the root is also illustrated in the dynamical planes: the lighter color, the faster convergence to the root. Let us remark that the increase of the maximum number of iterations does not change significantly the shape and details of the dynamical planes.

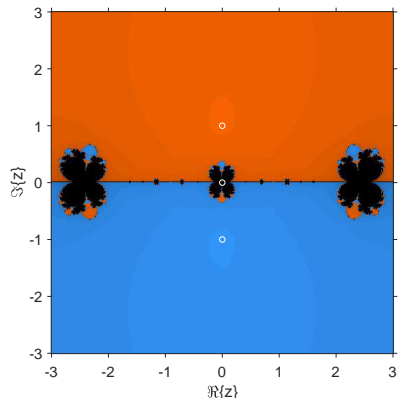

(a) $p_{1}(z)=\left(z^{2}+1\right)^{3}$

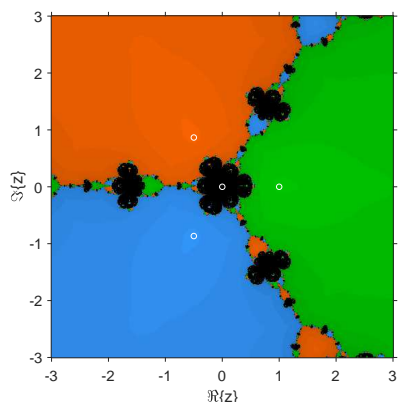

(c) $p_{3}(z)=\left(z^{3}-1\right)^{3}$

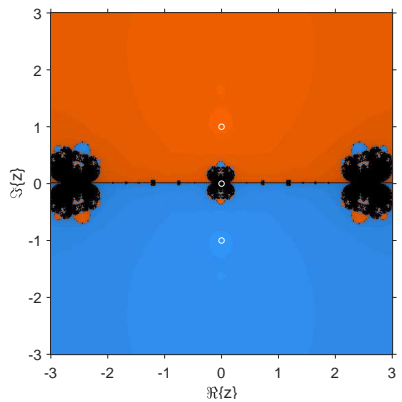

(b) $p_{2}(z)=\left(z^{2}+1\right)^{4}$

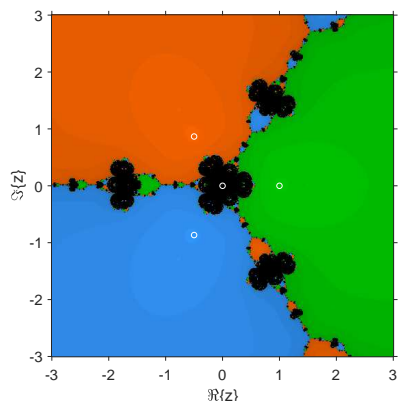

(d) $p_{4}(z)=\left(z^{3}-1\right)^{4}$

FiguRE 1. $M_{3}(z)$ dynamical planes for different polynomials in the square $[-3,3] \times[-3,3]$. 
For every dynamical plane of the different instances of $M_{3}(z)$, a wide convergence region can be found, since almost every initial guess tends to one of the roots of the corresponding polynomial.

Since 0 is, for every case, an indifferent fixed point, its behavior can not be determined previously, because it can operate either as an attracting or a repelling point. If a zoom is made on the origin, a one-point convergence to 0 can be found, as shown in Fig. 2. Despite the points in its neighborhood are black, they converge very slowly to 0 .

Finally, in the analyzed region no attraction to the infinity has been found.

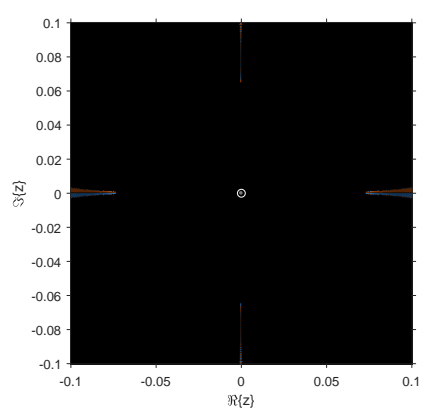

(a) $p_{1}(z)=\left(z^{2}+1\right)^{3}$

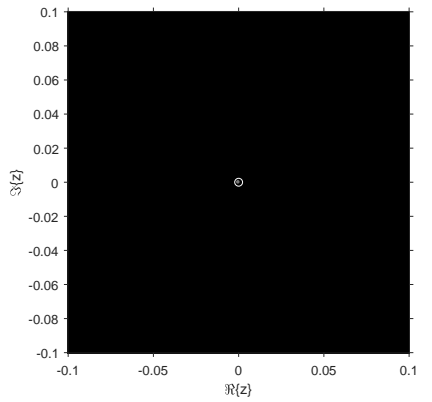

(b) $p_{4}(z)=\left(z^{3}-1\right)^{4}$

Figure 2. $M_{3}(z)$ dynamical planes for different polynomials in the square $[-0.1,0.1] \times[-0.1,0.1]$.

4.2.2. Dynamical analysis of the method $M_{4}(z)$. In order to compare the features of the methods $M_{3}(z)$ and $M_{4}(z)$, we are performing the dynamical analysis of $M_{4}(z)$ when it is applied on the same polynomials of the previous case.

The fixed point operator of $M_{4}(z)$ on $p_{1}(z)$ is

$$
M_{41}(z)=\left.M_{4}(z)\right|_{p_{1}(z)}=\frac{1+33 z^{2}+268 z^{4}+1641 z^{6}-395 z^{8}}{4 z+112 z^{3}+512 z^{5}-1396 z^{7}},
$$

whose fixed points are $z_{1,2}^{*}= \pm i, z_{3,4}^{F}= \pm i 0.2023, z_{5-8}^{F}= \pm 0.2375 \pm i 0.3160$. Computing $\left|M_{41}^{\prime}(z)\right|$, the roots $z_{1,2}^{*}$ are attracting, while the strange fixed points $z_{3-8}^{F}$ are repelling. The infinity is an attracting fixed point. As expected, $z_{1,2}^{*}$ are critical points, and there are 8 free critical points: $z_{3,4}^{C}=$ $\pm i 0.2590, z_{5,6}^{C}= \pm 0.2781$ and $z_{7-10}^{C}= \pm 0.0510 \pm 0.1865$.

Regarding to the application of $M_{4}(z)$ over $p_{2}(z)$, the fixed point operator is

$$
M_{42}(z)=\left.M_{4}(z)\right|_{p_{2}(z)}=\frac{1+73 z^{2}+1710 z^{4}+10750 z^{6}+51217 z^{8}-13191 z^{10}}{2 z+140 z^{3}+3000 z^{5}+12500 z^{7}-45658 z^{9}} .
$$


The fixed points of $M_{42}(z)$ are the roots $z_{1,2}^{*}= \pm i$ and 8 strange fixed points $z_{3-6}^{F}= \pm 0.2504 \pm i 0.3633$ and $z_{7-10}^{F}= \pm 0.0279 \pm i 0.1655$. Only the roots are superattracting, while the strange fixed points are repelling. The infinity is, again, an attracting fixed point. There are 12 free points out of 14 critical points, whose values are $z_{3,4}^{C}= \pm i 0.2342, z_{5,6}^{C}= \pm i 0.1839, z_{7,8}^{C}= \pm 0.3057$, $z_{9,10}^{C}= \pm i 0.1456$ and $z_{11-14}^{C}= \pm 0.0616 \pm i 0.1621$.

When $M_{4}(z)$ is applied on the polynomial $p_{3}(z)$, the fixed point operator gets into

$$
M_{43}(z)=\left.M_{4}(z)\right|_{p_{3}(z)}=\frac{N_{21}(z)}{D_{20}(z)},
$$

where $N_{21}(z)$ and $D_{20}(z)$ are polynomials of degree 21 and 20, respectively. The fixed points that match with the roots, $z_{1,2}^{*}=-\frac{1}{2} \pm i \frac{\sqrt{3}}{2}$ and $z_{3}^{*}=1$, are superattracting points. The 18 strange fixed points are repelling, and the infinity is an attracting fixed point. Regarding the critical points, 30 out of 33 are free.

Finally, applying $M_{4}(z)$ on the polynomial $p_{4}(z)$, the fixed point operator results

$$
M_{44}(z)=\left.M_{4}(z)\right|_{p_{4}(z)}=\frac{N_{27}(z)}{D_{26}(z)},
$$

where $N_{27}(z)$ and $D_{26}(z)$ are polynomials of degree 21 and 20, respectively. The fixed points of $M_{44}(z)$ are the roots of $p_{4}(z)$, whose behavior is superattracting, and 24 strange and repelling fixed points. The infinity is an attracting fixed point. There are 45 critical points, 42 of them are free.

Table 5 collects the information about the number of fixed and critical points of the previous methods. In a coarse comparison with Table 4, the number of free critical points match between both tables.

TABLE 5. Fixed and critical points of $M_{4}(z)$ on different polynomials

\begin{tabular}{|c|c|c|c|c|}
\hline$p(z)$ & $M(z)$ & $\begin{array}{c}\text { Number of } \\
\text { roots }\end{array}$ & $\begin{array}{c}\text { Number of strange } \\
\text { fixed points }\end{array}$ & $\begin{array}{c}\text { Number of free } \\
\text { critical points }\end{array}$ \\
\hline$p_{1}(z)=\left(z^{2}+1\right)^{3}$ & $M_{41}(z)$ & 2 & 6 & 8 \\
$p_{2}(z)=\left(z^{2}+1\right)^{4}$ & $M_{42}(z)$ & 2 & 8 & 12 \\
$p_{3}(z)=\left(z^{3}-1\right)^{3}$ & $M_{43}(z)$ & 3 & 18 & 30 \\
$p_{4}(z)=\left(z^{3}-1\right)^{4}$ & $M_{44}(z)$ & 3 & 24 & 42 \\
\hline
\end{tabular}

For the methods $M_{41}(z), M_{42}(z), M_{43}(z)$ and $M_{44}(z)$, the dynamical planes have been obtained, following the same routines than in the previous cases. However, in this case, only the fixed attracting points have been plotted in white circles. The dynamical planes are represented in Figure 3.

Once the dynamical planes have been represented, the main conclusion is that every initial guess converges to a superattracting fixed point. This fact is very important because it guarantees the stability of the iterative 


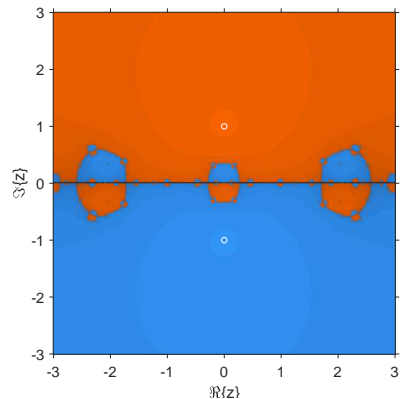

(a) $p_{1}(z)=\left(z^{2}+1\right)^{3}$

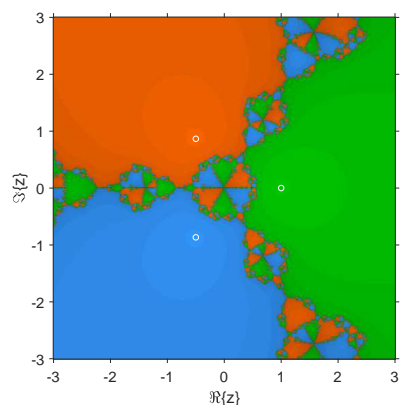

(c) $p_{3}(z)=\left(z^{3}-1\right)^{3}$

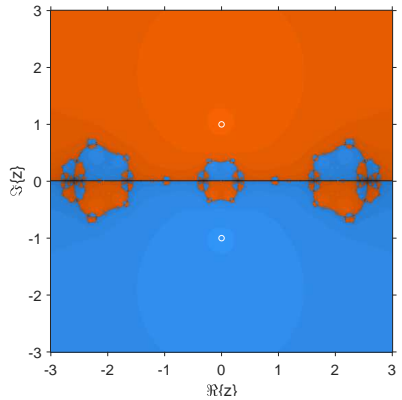

(b) $p_{2}(z)=\left(z^{2}+1\right)^{4}$

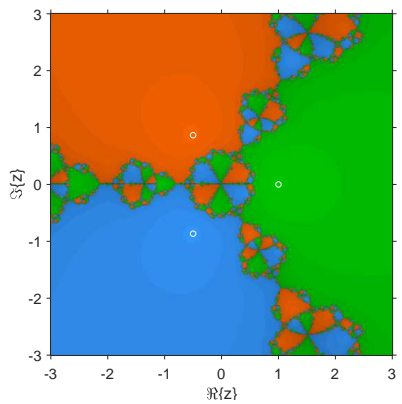

(d) $p_{4}(z)=\left(z^{3}-1\right)^{4}$

FiguRE 3. $M_{4}(z)$ dynamical planes for different polynomials

method for the cases that we have already studied. The absence of strange and attracting fixed points supports the wider basins of attraction.

The design of a non-optimal method of third order of convergence has resulted, by dynamical analysis, in a stable method with some areas that must be avoided. The evolution of this method, turned into an optimal method of order four, both has increased the order of convergence and has extended the basins of attraction, obtaining a full area of convergence for every point in the complex plane.

\section{References}

[1] D.S. Alexander, A History of Complex Dynamics: From Schroder to Fatou and Julia, Friedr \& Sohn Veriage, 1994.

[2] A.F. Beardon, Iteration of Rational Functions, Springer-Verlag, New York, 1991.

[3] P. Blanchard, The dynamics of Newton?s method, Proc. Sympos. Appl. Math., 49 (1994), 139-154.

[4] F. I. Chicharro, A. Cordero, J. R. Torregrosa, Drawing dynamical and parameters planes of iterative families and methods, Scientific World Journal, $\mathbf{7 8 0 1 5 3}$ (2013), 1-11. 
[5] C. Chun and B. Neta, A third order modification of Newton's method for multiple roots, Appl. Math. Compt., 211 (2009), 474-479.

[6] R. L. Devaney, An Introduction to Chaotic Dynamical Systems, Addison-Wesley publishing Company, New York, 1989.

[7] H.T. Kung and J.F. Traub, Optimal order of one-point and multipoint iterations, J. Assoc. Comput. Mach., 21 (1974), 643-651.

[8] B. Liu andX. Zhou, A new family of fourth order methods for multiple roots of non-linearr equation, Nonlinear Anal.: Model. Control, 18 (2013), 143-152.

[9] M.S. Petkovic, B. Neta, L.D Petcovic and J. Dzflnic, Multipoint Methods for Solving Nonlinear Equations, Elsevier, Waltham, MA, 2013.

[10] J. R. Sharma and R. Sharma, Modified Jarratt method for computing multiple roots , Appl. Math. Comp., 217 (2010), 878-881.

[11] J.L. Varona, Graphic and numerical comparison between iterative methods, Math. Intell. 24 (2002), 37-46.

[12] E.R. Vrscay and W.J. Gilbert, Extraneous fixed points, basin boundaries and chaotic dynamics for Schroder and Konig rational iteration functions, Numer. Math., 52 (1988), 1-16.

[13] S. Weerakoon and T. G. I. Fernando, A Variant of Newton's method with accelerated third-order convergence, Appl. Math. Lett , 17 (2000), 87-93.

Prem Bahadur Chand

Department of Mathematics

South Asian University

Akbar Bhawan, Chanakya Puri, New Delhi-110021, India

e-mail: premchand13@gmail.com

Francisco I. Chicharro

Escuela Superior de Ingeniería y Tecnología

Universidad Internacional de La Rioja

Av. La Paz 137, Logroño-26006, Spain

e-mail: francisco.chicharro@unir.net

Pankaj Jain

Department of Mathematics

South Asian University

Akbar Bhawan, Chanakya Puri, New Delhi-110021, India

e-mail: pankaj.jain@sau.ac.in

Kriti Sethi

Department of Mathematics

South Asian University

Akbar Bhawan, Chanakya Puri, New Delhi-110021, India

e-mail: kritisethi@students.sau.ac.in 